\title{
Primary Lymphoma of the Brain in a Young Man Whose Brother Died of Hemophagocytic Lymphohistiocytosis: Case Report
}

\author{
Eleonora Džoljić1,2*, Tatjana Stošić-Opinćal ${ }^{1,3}$, Milica Skender-Gazibara ${ }^{4}$ and Vladimir Kostić ${ }^{1,2}$ \\ ${ }^{1}$ University of Belgrade, School of Medicine, Belgrade, Serbia \\ ${ }^{2}$ Clinic of Neurology, Clinical Center of Serbia, Belgrade, Serbia \\ ${ }^{3}$ MR Centre, Clinical Center of Serbia, Belgrade, Serbia \\ ${ }^{4}$ Institute of Pathology, University of Belgrade, School of Medicine, Belgrade, Serbia \\ ${ }^{5}$ Clinic of Hematology, Clinical Center of Serbia, Belgrade, Serbia
}

Submission: December 18, 2018; Published: September 04, 2018

*Corresponding author: Eleonora Džoljić, Clinic of Neurology, University of Belgrade, School of Medicine, Belgrade, Serbia, Email: dr.elidz@gmail.com

Summary

Introduction We represent the unique occurrence of primary central nervous system lymphoma (PCNSL) in a patient whose brother died of genetically confirmed hemophagocytic lymphohistiocytosis (HLH). Case Outline We report a case of a 25-year-old male patient with primary aggressive diffuse large B-cell lymphoma affecting the brain and PCNSL. Despite one year of medical treatment outcome was lethal. However, our patient had a relatively longer survival compared to median survival time for PCNSL. Additionally, he had two older brothers who died at the age of about 11 years. One died of fulminate malignancy, shortly after pediatric admission, before the diagnosis could be established. The other one died from genetically confirmed (perforin mutation/PRF1) HLH. Our patient was heterozygous carrier of perforin mutation representing the genetic marker for HLH. Our patient's father was the carrier of the same mutation but had no symptoms of any disease. Conclusion This case points at the presence of HLH and diffuse large B-cell PCNSL in brothers. Extensive assessment of patients with probable PCNSL and familial HLH is necessary, including genetic analysis for HLH.

Keywords: Primary aggressive diffuse large B-cell CNS lymphoma; Hemophagocytic lymphohistiocytosis; Perforin mutation

Abbreviations: PCNSL: Primary Central Nervous System Lymphoma; HLH: Hemophagocytic Lympho Histiocytosis; CNS: Central Nervous System; BBB: Blood-Brain Barrier; DLBCL: Diffuse Large B-Cell Lymphomas; IgE: immunoglobulin E; MRIs: Magnetic Resonance Imaging; cMRI: Conventional Magnetic Resonance Imaging

\section{Introduction}

Primary Central Nervous System Lymphoma (PCNSL) is a rare tumor that afflicts approximately 1,000 people in the United States each year. PCNSL has captured the attention of neurooncologists for several reasons. First, it arises from a cell type not normally present in the Central Nervous System (CNS). Second, it can completely regress with either corticosteroids or cranial irradiation, it is recurrent and like glioblastoma multiforme it has a rapidly fatal course killing most patients within a year [1]. Third, the Blood-Brain Barrier (BBB) is critically important because much of the tumor resides behind an intact BBB and often cannot be visualized radiographically [2]. About $90 \%$ of PCNSLs are aggressive Diffuse Large B-Cell Lymphomas (DLBCL) of histopathological type [3]. It represents the most commonly occurring lymphoid malignancy [4]. DLBCL represents about 4\% of CNS tumors, and 1\% of all non-Hodgkin lymphomas [4].
DLBCL is a typical presentation of PCNSL in immunocompetent patients with progressive focal symptoms and mass lesions [1]. However, PCNSL has a different gene expression signature from that of systemic DLBCL [5]. Hemophagocytic LymphoHistiocytosis (HLH) is an uncommon hematologic disorder. It is a life-threatening disease of severe inflammation syndrome caused by uncontrolled proliferation of activated lymphocyte/macrophage system characterized by proliferation of benign lymphocytes and macrophages that secrete high amounts of proinflammatory cytokines. It is classified as cytokine over-secretion syndrome or one of cytokine storm syndromes [6]. The aim of this case report is representation of the unique occurrence of PCNSL in a patient whose brother died of genetically confirmed HLH. 


\section{Open Access Journal of Neurology \& Neurosurgery}

\section{Case Report}

\section{Clinical presentation}

A previously healthy 25-year-old man with subacutely developed clinical and MRI signs of multifocal CNS lesions was admitted at the Neurology Clinic in Belgrade, Clinical Center of Serbia. Neurological examination revealed ataxia with bilateral pyramidal signs, elevated blood pressure 140/110 mmHg and signs of brain stem dysfunction at auditory and somato-sensory evoked potentials. Routine biochemical and hematological findings were normal. HIV status was negative. Additionally, our patient had two older brothers who died at the age of about 11 years. One died of fulminate malignancy, shortly after pediatric admission, before the diagnosis could be established. The other one died of genetically confirmed HLH. Therefore, genetic testing of our patient's blood was made for HLH, X-linked lymphoproliferative syndrome (perforin, syntaxin, MUNC 13-4, XLP-1, XLP-2) of neurofibromatosis type 1 (NF1), since MRI of the brain revealed the suspected features of NF1.

Genetic tests were negative except for a heterozygous mutation type 1 in the perforin gene (g.PRF1/p. A437V). Our patient's father was the carrier of the same mutation and he had no symptoms of any disease. In accordance, additional extensive analyses were performed in our patient. However, during the first 11 months of the disease course, except for a slightly elevated protein level in the CerebroSpinal Fluid (CSF) 0.84 $\mathrm{g} / \mathrm{L}$ ), all results were within the normal range: CSF examination was without oligoclonal bands, CSF sediment analysis as well as biochemical CSF and blood examinations (including angiotensin converting enzyme), immunological, hematological (including bone marrow biopsy) and hormonal analyses of blood, as well as cultures of blood, urine and CSF, all were negative and accompanied with negative antibodies in CSF and blood for relevant bacterial, viral and fungal infections. Ten months after the first signs of neurological disease pathological findings were as follows: slight splenomegaly by abdominal echosonography and prominent left and right hilum by chest X-ray, as well as elevated blood levels of immunoglobulin E (IgE), cysteine-rich protein type 2 (CRP2) and beta-2-microglobulin (b2M). Multiple magnetic resonance imaging (MRIs) of the brain were performed and only the last MRI, scanned a month before death, a year from the onset of the disease, revealed signs of brain lymphomatosis with focal brain lesions.

MR findings Conventional Magnetic Resonance Imaging (cMRI) was performed in axial, coronary and sagital planes in T2W, FLAIR-T2W, T1W pre and postcontrast sequences with diffusion-weighted imaging and single-voxel magnetic resonance spectroscopy with short echo time (30 ms). cMRI showed multiple focal lesions, mostly punctiform (1-3 $\mathrm{mm}$ in diameter), localized diffusely along the perivascular space and corticomedullar border, as well as in the deep white matter of the cerebral hemispheres and periventricular space, in the basal ganglia and corpus callosum. Lesions were T2W and FLAIR hyperintense, T1W hypointense and showed nodular postcontrast enhancement with restricted diffusion. Identical lesions were seen in the brain stem, cerebellum and cervical spinal cord at the C1-C4 level. Dural and leptomeningeal postcontrast enhancement were also seen (Figure 1).

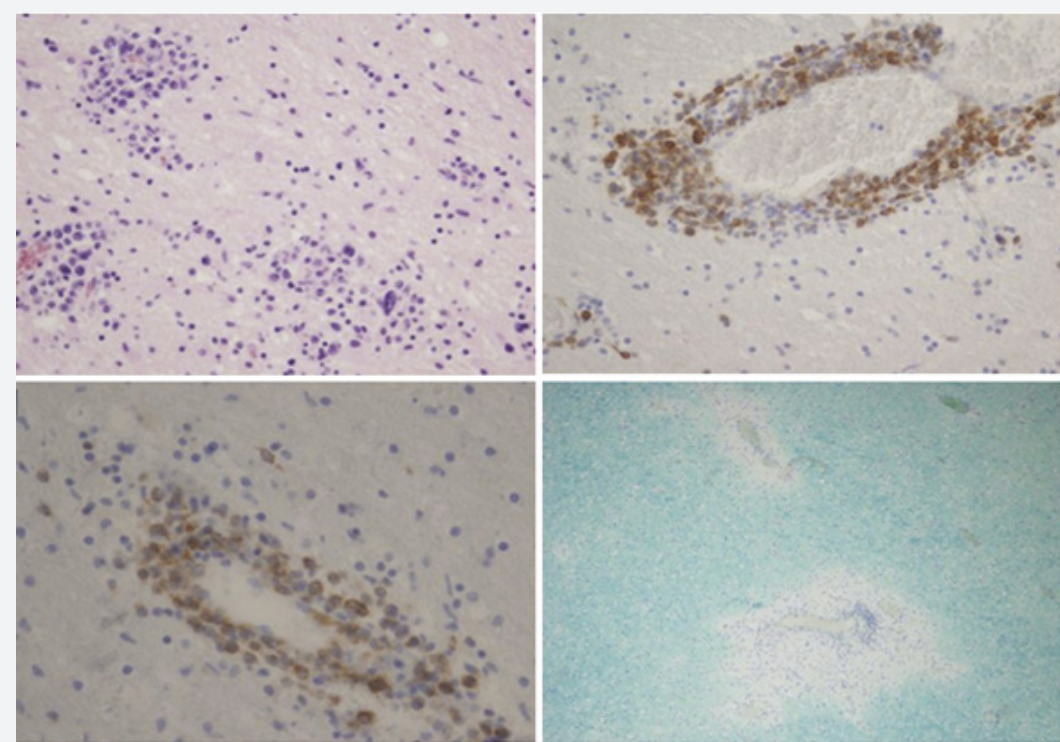

Figure 1: Sagittal T2W (A), coronal FLAIR (B) and coronal T1-weighed contrast-enhanced (C) MR images of a patient with PCNSL show multiple punctiform lesions of the corpus callosum, basal ganglia and white matter of the brain and cervical spine

Voxel placed in parietal subcortical lesions showed normal values of NAA/Cr (2.13), slightly elevated Cho/Cr ratio (1.12) and the presence of amino acids peaks. Differential diagnosis included infection (viruses, fungi), neoplastic lesions (intravascular lymphoma), eosinophilic vasculitis, and granulomatosis (sarcoidosis). Therapy Ten months of applied medicament therapy, according to clinical presentation and MR findings, included neurological and later hematological corticosteroid therapy which produced partial and transient short lasting remissions. However, the patient's parents refused 


\section{Open Access Journal of Neurology \& Neurosurgery}

biopsy of the patient's leptomeninges or brain. Neurological therapy consisted of corticosteroid application, intravenously (i.v) pulse methyl-prednisolone therapy of $1000 \mathrm{mg}$ per day in 5 days duration with 3 weeks to month resting intervals, with following oral prednisolone medication (doses depended on the severity of clinical presentation). Important observation was that any discontinuation of corticosteroid therapy coincided with the occurrence of CNS relapse.

Later, dexasone was administered short lastingly in the intramuscular dose of 8-16 mg, depending on intracranial pressure upraise severity. Since at last month of the patient's life he had high fever with the signs of systemic inflammation, the patient was admitted at the Clinic of Hematology shortly before his death. Unfortunately, in spite of all diagnostic and therapeutic procedures, one year from the onset of the disease the patient died. Autopsy findings - neuropathological analysis. The brain (weighted 1,400 grams) and spinal cord were fixed in $10 \%$ buffered formalin. Representative tissue samples from the brain hemispheres, brain stem, cerebellum and spinal cord were embedded in paraffin and $5 \mu \mathrm{m}$ thick sections were stained with hematoxylin-eosin and Luxol fast blue. Immunohistochemistry was performed using following antibodies (DAKO): CD20, CD79a, BCL-2, CD3, TdT, CD34, MPO, CD68, GFAP and CD30. Grossly, on the frontal sections of the brain, the best visible grayish foci of discoloration were present in the left part of the corpus callosum and ipsilateral internal capsule as well as in the sections of the distal segments of cervical spinal cord. Histopathological examination showed multifocal, typical angiocentric infiltration with lymphoid cells, large vesicular nuclei and distinct nucleoli (centroblasts and immunoblasts) admixed with some small lymphocytes and macrophages (Figure 2A).

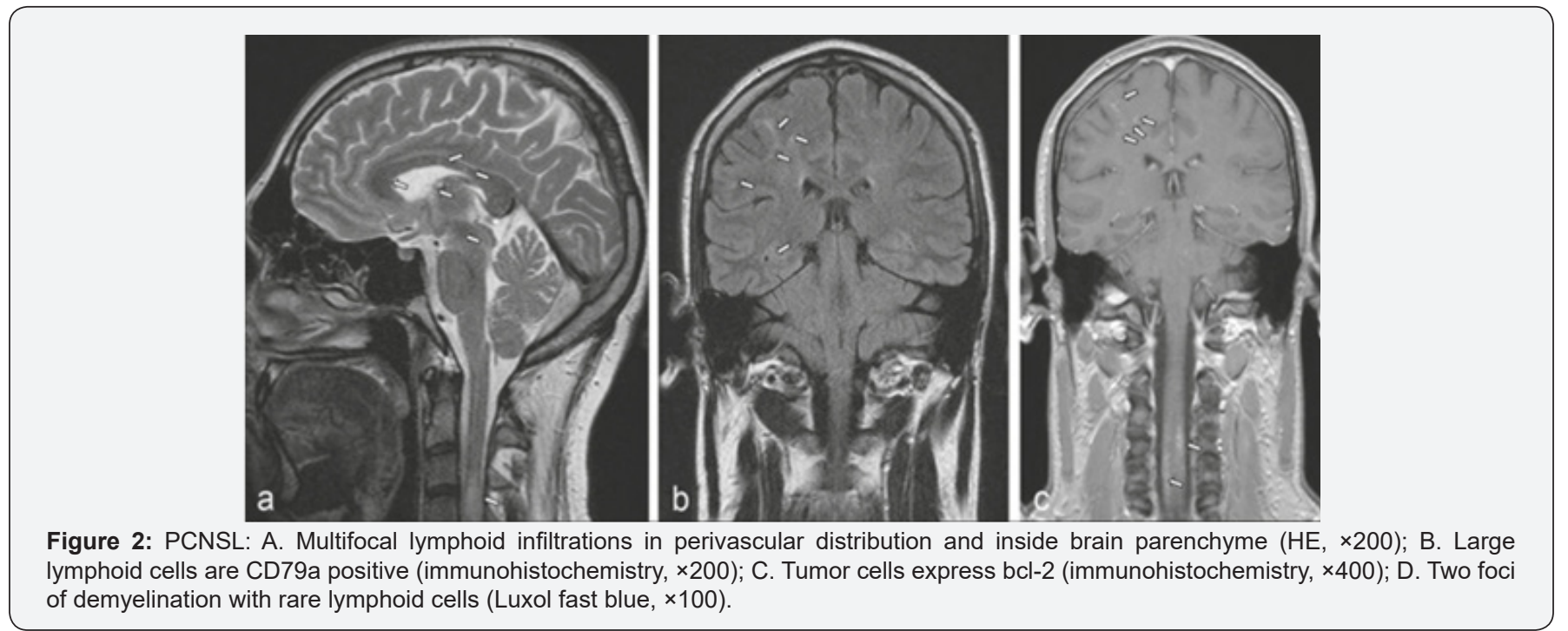

Large lymphoid cells expressed B cell markers (CD20, CD79a) and BCL-2 (Figures 2B and 2C). Beside tumor cell infiltrations, there were many small foci of demyelization (Figure 2D) (sentinel lesions) which were pronounced in sections of the cerebellum and cervical spinal cord. General autopsy examination did not recognize any malignancy outside the CNS. The cause of death was Aspergillus infection of the lung with infiltration of pulmonary blood vessels and their thrombosis which was a direct death-leading event and the direct cause of death. Final diagnosis was PCNSL-diffuse large-B cell non-Hodgkin lymphoma. DISCUSSION The most important of our findings is unique occurrence of PCNSL in the family with genetically confirmed HLH.

Recently, some associations of HLH with B-cell lymphoproliferative disorder has been reported [7-11], but only this case report describes primary brain lymphoma in a young man whose brother died of HLH. Outcome of patients with relapse of PCNSL is generally poor, with the median survival time of 2-5 months [12]. Nevertheless, our patient had several incomplete remissions followed with a longer total survival time, approximately 1 year. One of possible explanations could be the occurrence of specific lymphoma subtype, but still no significant correlation was shown between specific lymphoma subtypes and the patient's prognosis [13]. Some data correlated the disease prognosis with specific therapy, including postoperative radiotherapy and chemotherapy [13]. Our patient was treated mainly with corticosteroids, later on with hematologic chemotherapy and it was possible only approximately to compare his disease outcome with other similar ones [13].

Recent results have shown the efficacy of corticosteroid therapy in the prevention of disease relapse [14,15]. This procedure usually forms an integral part of hematologic treatment protocols for acute lymphoblastic leukemia, lymphoblastic and Burkitt's lymphomas. Neurological relapse rates are $30-50 \%$ for these three diseases, and corticosteroid therapy has been shown to unequivocally reduce the occurrence of CNS relapse [16]. Furthermore, new data have shown a longer-term survival in patients with diagnosed PCNSL treated with corticosteroids combined with chemotherapy and wholebrain radiation therapy [17]. Consistent with these results 


\section{Open Access Journal of Neurology \& Neurosurgery}

$[16,18]$, treatment with combination of corticosteroid as and hematologic chemotherapy might be the one of potential explanations for a relatively longer survival time in our patient. Additionally, our patient's lymphoma cells were BCL-2 positive. New studies have appointed that BCL-2 positive PCNSL occurs in about $46.8 \%$ of cases [13], while phenotype of PCNSL is usually characterized by BCL-6 expression [19]. Our patient's lymphoma cells were $\mathrm{CD} 79 \alpha$ positive as well.

It has been shown that PCNSL could originate from late germinal B cells center $[20,21]$, which is consistent with findings of CD79 $\alpha$ positive PCNSL cells [17]. In contrast to previous [13], recent results have shown the significance of specific BCL-2 and BCL- 6 phenotype as the indicator of short progression and free survival [22]. Moreover, the main lymphoma baseline characteristics were analyzed in 65 patients with PCNSL [23]. Accordingly, our patient represents a rare coexistence of uncommonly affected locations including not only brain but also leptomeninges (7\%) and spinal cord (1\%). In addition, the normal range or elevated protein levels in CSF, as in our case, are also characteristics of PCNSL [24]. The most important issue we need to address here is the unique occurrence of PCNSL and HLH among brothers. But, could there be any connection between PCNSL and HLH? Our patient did not suffer from HLH as he only had a genetically confirmed heterozygous PRF1/FHL2 mutation. It represents one of the markers of confirmed HLH in homozygous state, which was not the case in our patient [25].

Recently, Trapani et al. [26] have shown that the partial inactivation of PRF1 due to mutations that promote protein misfolding or a common hypomorphic allele coding have been associated with lymphoid malignancies in childhood, adolescence as well as in adulthood. In their study, adult patients with PRF1/ A91V mutation secondary developed B-cell lymphoma. However, our patient had mutation PRF1/A437V also inactivating gene mutation. This might suggests a similar relation of heterozygous mutation/partial inactivation of PRF1 gene with PCNSL and thus indirectly indicates some connection between HLH and PCNSL. Thus, for patients with partially active PRF1 alleles, due to any reason, might be expected to develop secondary lymphoma or melanoma but not HLH as they do not express complete PRF1 inactivation i.e. a homozygous one (bi-allelic) necessary for HLH. Our patient had several severe predictors of short survival; not only PRF1 mutation (p.A437V) but also a subacute progressive form of the disease and a poor prognosis predictor phenotype [22]. Nevertheless, our patient had a relatively longer survival compared to median survival time for PCNSL [12].

It can be concluded that the presence of particular mutation of perforin gene might be considered as the probable genetic basis for the familiar appearance of lymphoproliferative disease as well as other malignant disease of unknown origin. Also, there is a possibility that additional genetic defect exists in the family. Thus, extensive assessment of the patient with probable PCNSL in the family with HLH is necessary.

\section{Acknowledgement}

This work was supported by the Ministry of Education, Science and Technological Development of the Republic of Serbia, through the Contract No. 175090 (2011-2014). Additionally, I would like to thank Dr Ivan Grbatinić for significant contribution he has given to this manuscript.

\section{References}

1. Gallop-Evans E (2012) Primary central nervous system lymphoma. ClinOncol (R Coll Radiol). 24(5): 329-338.

2. Lai R, Rosenblum Mk, DeAngelis LM (2002) Primary CNS lymphoma: A whole brain disease? Neurology 59(10): 1557-1562.

3. Montesinos-Rongen M, Siebert R, Deckert M (2009) Primary lymphoma of the central nervous system: just DLBCL or not? Blood 113(1): 7-10.

4. Nastoupil LJ, Rose AC, Flowers CR (2012) Diffuse large B-cell lymphoma: current treatment approaches. Oncology (Williston Park)26: 488-495.

5. Rubenstein JL, Fridlyand J, Shen A, Aldape K, Ginzinger D, et al. (2006) Gene expression and angiotropism in primary CNS lymphoma. Blood. 107: 3716-323.

6. Fisman DN (2000) Hemophagocytic syndromes and infection. Emerg Infect Dis 6(6): 601-608.

7. Aggarwal D, Gupta R, Singh S, Gupta K, Kudesia M (2010) Hemophagocytic lymphohistiocytosis in B-cell lymphoproliferative disorder: Report of a rare association. Indian J Hematol Blood Transfus 26(2): 74-76.

8. Balwierz W, Czogała M, Czepko E (2010) Anaplastic large cell lymphoma associated with hemophagocytic lymphohistiocytosis: a case report and review of the literature. Przegl Lek 67(6): 436-438.

9. Cwiklińska M, Czogała M, Balwierz W, Hnatko-Kołacz M, MorylBujakowska A, et al (2010) Hemophagocytic syndrome in children with different underlying conditions. Przegl Lek 67 (6): 430435 .

10. Nakamura S, Murase T, Kinoshita T (2007) Intravascular large B-cell lymphoma: the heterogeneous clinical manifestations of its classical and hemophagocytosis-related forms. Haematologica 92(4): 434-436.

11. Altaf A, Arteaga GM, Joshi AY, Rodriguez V (2012) Diffuse large B-cell lymphoma in an adolescent female presenting with Epstein-Barr virusdriven hemophagocytic lymphohistiocytosis: a case report. J Med Case Rep 6:141.

12. Kridel R, Dietrich PY (2011) Prevention of CNS relapse in diffuse large B-cell lymphoma. Lancet Oncol 12(13): 1258-1266.

13. Min M, Lin L, Bi CF, Wang XQ et al. (2012) Analysis of the immunohistochemical subtypes and prognosis of primary diffuse large B cell lymphoma of the central nervous system. ZhonghuaZhong Liu ZaZhi 34(2): 110-116.

14. Hill Q Owen R (2006) CNS prophylaxis in lymphoma: who to target and what therapy to use. Blood Rev 20(6): 319-332.

15. Kwong Y, Yeung D, Chan J (2009) Intrathecal chemotherapy for hematologic malignancies: drugs and toxicities. Ann Hematol 88(3): 193-201.

16. Blum K, Lozanski G, Byrd J (2004) Adult Burkitt leukemia and lymphoma. Blood 104(10): 3009-3020.

17. Deckert M, Paulus W (2007) Malignant lymphomas. In: Louis DN, et al. (Eds) WHO Classification of Tumours of the Central Nervous System. Lyon, France: Wiestler\& W. K. Cavenee, IARC Pp.188-192.

18. Motomura K, Natsume A, Fujii M, Ito M, Momota H, et al. (2011) 


\section{Open Access Journal of Neurology \& Neurosurgery}

Long-term survival in patients with newly diagnosed primary central nervous system lymphoma treated with dexamethasone, etoposide, ifosfamide and carboplatin chemotherapy and wholebrain radiation therapy. Leuk Lymphoma 52(11): 2069-2075.

19. Deckert MA, Engert A, Brück W, Ferreri AJ, Finke J, et al. (2011) Modern concepts in the biology, diagnosis, differential diagnosis and treatment of primary central nervous system lymphoma. Leukemia. 25(12) 1797-17807.

20. Montesinos-Rongen M, Brunn A, Bentink S, Basso K, Lim WK, et al. (2008) Gene expression profiling suggests primary central nervous system lymphomas to be derived from a late germinal center B cell Leukemia 22(2): 400-405.

21. Baraniskin A, Deckert D, Schulte-Altedorneburg G, Schlegel U, Schroers $\mathrm{R}$ (2011) Current strategies in the diagnosis of diffuse large B-cell lymphoma of the central nervous system. Br J Haematol 156(4): 421432.

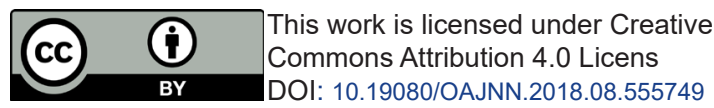

22. Maeshima AM, Taniguchi H, Fukuhara S, Morikawa N, Munakata W, et al. (2012) Bcl-2, Bcl-6, and IPI are prognostic indicators in patients with DLBCL treated with rituximab-containing chemotherapy. Cancer Sci 103(10): 1898-1904.

23. Kim JE, Yoon DH, Kim S, Lee DH, Kim JH, et al. (2012) Relapse pattern and prognostic factors for patients with primary central nervous system lymphoma. Korean J Hematol 47(1): 60-66.

24. Ferreri AJM, Marturano E (2012) Primary CNS lymphoma. Best Practice \& Research Clinical Haematology 25(1): 119-130.

25. Zhang K, Filipovich AH, Johnson J, Marsh RA, Villanueva J (2013) Hemophagocytic lymphohistiocytosis, familial. In: Pagon RA, et al. (Eds) In: Seattle and National Center for Biotechnology Information. GeneReviews ${ }^{\circledR}$. Seattle, WA: University of Washington,USA.

26. Trapani JA, Thia KY, Andrews M, Davis ID, Gedye C, et al. (2013) Human perforin mutations and susceptibility to multiple primary cancers. Oncoimmunology 2(4): e24185.

Your next submission with Juniper Publishers
will reach you the below assets
- Quality Editorial service
- Swift Peer Review
- Reprints availability
- E-prints Service
- Manuscript Podcast for convenient understanding
- Global attainment for your research
- Manuscript accessibility in different formats
( Pdf, E-pub, Full Text, Audio)
- Unceasing customer service
Track the below URL for one-step submission
https://juniperpublishers.com/online-submission.php

